\title{
CHEERS to 30 Years of PharmacoEconomics
}

\author{
Richard Milne ${ }^{1} \cdot$ Christopher Carswell ${ }^{2}$
}

Accepted: 3 November 2021 / Published online: 18 December 2021

(c) The Author(s), under exclusive licence to Springer Nature Switzerland AG 2021

PharmacoEconomics celebrates 30 years of publishing this year. The journal was launched as an Adis journal in January 1992 and was the first international peer-reviewed journal to exclusively promote and publish research on the practical application of economics to pharmacotherapy. Originally intended as a monthly subscription review journal, it also accepted research articles as there was insufficient research to provide enough material for review articles. Today, the journal continues to publish both reviews and original research.

The first editorial reported that 700 articles containing the terms 'drugs' and 'economics' had been published between 1966 and 1990 [1]. In October 2021, there were almost 22,000 articles within this classification in MEDLINE alone. However, adding 'cost effectiveness' as a keyword expanded this to 1.5 million articles, and including 'quality of life' added another 330,000. The field has broadened since the early days and now covers analytical methods, pharmaceuticals, vaccines, medical devices, and aspects of health policy, as evidenced by the current scope of PharmacoEconomics. There were over 400,000 downloads of the journal's articles in 2020, and the journal has a current 5-year impact factor of 5.733 .

Like all sciences, the discipline of pharmacoeconomics was initially a collective human activity of the privileged, favouring affluent countries with well-established centres of learning, government, and industry. This suggests why articles in the journal have predominantly been from the UK, USA, and Western Europe, accounting for three-quarters of the papers published in 2020. Although the journal has published relatively fewer articles from Eastern Europe, Asia, the Indian subcontinent, South America, Africa, and Oceania, we do see this gradually changing. We might even see

Richard Milne

richard.milne@hoa.co.nz

1 Health Outcomes Associates Limited and University of Auckland, Auckland, New Zealand

2 Adis Journals, Springer Nature, Auckland, New Zealand an article on the economics of healthcare during wintering over in Antarctica. The Professional Society for Health Economics and Outcomes Research (better known as ISPOR) has over 80 regional chapters across all regions of the world, reflecting the global interest and use of health economics (https://www.ispor.org/member-groups/global-groups/regio nal-chapters). One of our most popular themed issues was devoted to developing nations. In parallel with this, we would also like to further diversify our editorial board and attract members from Africa, Asia, and South America.

A key component in the success of any journal is the quality of the editorial board. We would like to take this opportunity to sincerely thank all those who have served on the board for their hard work and dedication to the journal. In particular, we would like to note four members of the current editorial board who have served since the beginning: Lyle Bootman, Mike Drummond, Silvio Garattini, and Richard Milne. We would also like to commemorate those members of the first editorial board who have died, including Alan Maynard, who visited NZ in 1991 to advise the editor; Michael Cooper; John Eisenberg, who wrote the first editorial [1]; and Alan Hillman.

In 30 years of publishing, there have been changes in topics and emphasis as many more countries require costeffectiveness analysis to inform decisions regarding reimbursement of healthcare interventions. Cardiovascular disease, antibiotic therapy, and antidepressants were some of the initial targets for economic analysis in the journal. Many of the earlier studies were simple cost analyses, assessments of resource utilisation and humanistic burden, economic evaluations alongside clinical studies with small sample sizes (often retrospective chart reviews), and simple models with limited deterministic sensitivity analysis. However, we soon published the first Markov model with a lifetime horizon [2], followed in 1998 by the seminal article on Markov modelling [3]. The development of computer technology has allowed the field to become ever more complex and sophisticated, with increasing reliance on statistical methods (such as value-of-information methods and econometrics) and complex models such as discrete-event 
simulation. Stochastic sensitivity analysis has become the norm for cost-effectiveness analysis but not yet for budget impact or cost analysis [4].

Pharmacoeconomic analysis is best suited to costly interventions and/or therapies for prevalent diseases, both of which have the potential for high budget impact to the payer and high market value to the supplier of a medicine, vaccine, or medical device. Economic evaluation is also important in policy development. Topics that have generated high interest from readers include methodology, chronic diseases (e.g. diabetes, cardiovascular disease, rheumatoid arthritis, and osteoporosis), and oncology. Atrial fibrillation and chronic obstructive pulmonary disease, which are both prevalent and costly, have received less attention and await new therapies. Medical devices and vaccines have also been less popular topics. However, the coronavirus disease 2019 (COVID-19) pandemic has given rise to a number of economic studies on vaccines and diagnostic testing, and we can expect many more.

Quality-adjusted life-years (QALYs)—despite initial challenges in their development and unresolved issues such as the trade-off between quantity and quality of life-have become widely used in economic evaluation. A whole new industry of preference-based measures of health has emerged, including generic and condition-specific measures and mapping techniques, and the journal receives a large number of papers in this area. Other topics on which we receive appreciable numbers of papers include cost effectiveness of interventions in oncology and rare diseases, caregiver burden, threshold derivation, and addressing decision uncertainty.

Although the field of pharmacoeconomics and outcomes research has seen extraordinary development over the past 30 years, some issues raised in an article in the very first issue of the journal remain. These include the potential for bias in economic evaluations, with many studies still sponsored/conducted by the pharmaceutical industry and consultancies; lack of head-to-head data; failure to publish negative findings and study protocols; and the continuing need to educate healthcare professionals on how to interpret economic evidence [5].

Alongside the development of digital technology and the increased sophistication of pharmacoeconomic methods, the publishing industry has undergone significant transformation, including a gradual move away from a primarily printbased subscription model towards an open access digital ecosystem. As word limits become less important and journals develop the capacity to host large amounts of data, there is increasing emphasis on improving transparency and on the replication of the results of published papers, alongside a move towards publishing economic models [6]. Journals are also increasingly adapting to using social media, publishing supplementary digital material (videos, podcasts, etc.), and engaging patients to help disseminate and improve implementation of research. This is increasingly important given that the transformation of the publishing industry has enabled an explosion of journal titles and published literature, making it more challenging to sort the wheat from the chaff. We would like to think that, over the last 30 years of publishing, PharmacoEconomics has played a pivotal role in helping all decision makers, whether patients, caregivers, physicians, or payers, in this regard.

We would like to express our heartfelt thanks to all our authors, reviewers, editorial board members, and readers who have made the journal what it is today. We hope you are proud of the journal, and we look forward to the next 30 years!

Richard J. Milne $\mathrm{PhD}$ (founding Editor) Chris Carswell MSc (current Co-Editor in Chief)

Funding No funding was received for the preparation of this editorial.

\section{Declarations}

Conflict of interest Richard Milne was previously an editor of PharmacoEconomics and is currently on the editorial board of the journal. Chris Carswell is the current Co-Editor in Chief of the journal and an employee of the publisher, Springer Nature.

\section{References}

1. Eisenberg JM. Why a journal of pharmacoeconomics? Pharmacoeconomics. 1992;1(1):2-4.

2. Hatziandreu EJ, Brown RE, Revicki DA, Turner R, Martindale J, Levine $S$, et al. Cost utility of maintenance treatment of recurrent depression with sertraline versus episodic treatment with dothiepin. Pharmacoeconomics. 1994;5(3):249-68.

3. Briggs A, Sculpher M. An introduction to Markov modelling for economic evaluation. Pharmacoeconomics. 1998;13(4):397-409.

4. Husereau D, Drummond M, Petrou S, Carswell C, Moher D, Greenberg D, et al. Consolidated Health Economic Evaluation Reporting Standards (CHEERS) statement. Pharmacoeconomics. 2013;31(5):361-7.

5. Drummond MF. Economic evaluation of pharmaceuticals: science or marketing? Pharmacoeconomics. 1992;1(1):8-13.

6. Husereau D, et al. Consolidated Health Economic Evaluation Reporting Standard 2022 (CHEERS 2022) statement: updated reporting guidance for health economic evaluations. BMJ. 2022 (in press) 